\title{
A new UK fission yield evaluation UKFY3.7
}

\author{
Robert William Mills ${ }^{\mathrm{a}}$ \\ The UK National Nuclear Laboratory, Central Laboratory, Sellafield, Seascale, Cumbria, UK
}

\begin{abstract}
The JEFF neutron induced and spontaneous fission product yield evaluation is currently unchanged from JEFF-3.1.1, also known by its UK designation UKFY3.6A. It is based upon experimental data combined with empirically fitted mass, charge and isomeric state models which are then adjusted within the experimental and model uncertainties to conform to the physical constraints of the fission process. A new evaluation has been prepared for JEFF, called UKFY3.7, that incorporates new experimental data and replaces the current empirical models (multi-Gaussian fits of mass distribution and Wahl Zp model for charge distribution combined with parameter extrapolation), with predictions from GEF. The GEF model has the advantage that one set of parameters allows the prediction of many different fissioning nuclides at different excitation energies unlike previous models where each fissioning nuclide at a specific excitation energy had to be fitted individually to the relevant experimental data. The new UKFY3.7 evaluation, submitted for testing as part of JEFF-3.3, is described alongside initial results of testing. In addition, initial ideas for future developments allowing inclusion of new measurements types and changing from any neutron spectrum type to true neutron energy dependence are discussed. Also, a method is proposed to propagate uncertainties of fission product yields based upon the experimental data that underlies the fission yield evaluation. The covariance terms being determined from the evaluated cumulative and independent yields combined with the experimental uncertainties on the cumulative yield measurements.
\end{abstract}

\section{Introduction}

Reliable and complete libraries of fission product yields with specified accuracies are needed for many different nuclear reactor calculations, including those on decay heat, dosimetry, burn-up, fuel handling, nuclide inventory and safety.

This paper describes the latest such UK evaluation of fission yields. In the UK the evaluation of yields for use in computer libraries was pioneered by Crouch at Harwell [1]. The work was then continued at Winfrith, producing $\mathrm{C} 4 \mathrm{U}$ and $\mathrm{C} 4 \mathrm{~A}$ known as UKFY1 [2], which were submitted to the first stage, JEF1, of the Joint Evaluated File. UKFY2 [3] and UKFY3.6A [4,5] followed resulting in the JEF-2.2 and JEFF-3.1.1 fission yield libraries.

The new library, UKFY3.7, which has been submitted to the JEFF project for testing, builds upon the earlier libraries. Improvements include bringing the database of fission yield measurements up to date by including 11 newly published measurements and using the GEF model [6] to predict unmeasured mass and charge yield distributions for all fissioning systems rather than fitting the experimental data to a combination of semi-empirical models for each system (multi-Gaussian fits of mass distribution and Wahl Zp model for charge distribution). The evaluation procedure is described and the effects of the changes from JEFF-3.1.1 studied.

Initial testing of the UKFY3.7 library, using the JEFF3.1.1 decay data is described for calculation of delayed neutron emission per fission, fission product decay heat pulse benchmarks and spent fuel decay heat.

The possible future developments of the fission yield libraries are then discussed. These include; (i) production of covariance terms so that useful uncertainties can be determined on fission product inventories, (ii) including non-traditional fission product yield measurements in evaluations and (iii) changing the fission product yield evaluations from a typical standard neutron spectra to an energy dependent description.

\section{The UKFY3.7 evaluation}

The production of the UKFY3.7 library is based upon 6 steps described previously [4];

- Statistical analysis of all available experimental data producing a recommended value and uncertainty for all measured yields.

- For all important fissioning system, see Table 1, fill all missing mass distributions and independent yields (including the ternary yields) using an appropriate model that estimates both the value and it uncertainty.

- Adjust the independent yields for each fissioning system to minimise the differences for each physical constraint such as conservation of mass and charge, and ensuring that complement element yields are equal.

- Split the independent yields using the isomeric splitting ratios based upon the Madland and England model.

- Calculate cumulative yields using the independent yields and JEFF-3.1.1 decay data. Then using the

\footnotetext{
a e-mail: robert.w.mills@nnl.co.uk
}

(c) The Authors, published by EDP Sciences. This is an Open Access article distributed under the terms of the Creative Commons Attribution License 4.0 (http://creativecommons.org/licenses/by/4.0/). 
Table 1. Fissioning systems in the UKFY3.7 that were deemed important in previous work based upon the maximum fraction of fissions in UOX, MOX and thorium fuels in fast and thermal reactors [4] and, following a user request, U236 thermal fission.

\begin{tabular}{|c|c|c|c|}
\hline \multicolumn{3}{|c|}{ Max. Fraction of Fission Rate } & \multirow[b]{2}{*}{ Spont. fission } \\
\hline$>10 \%$ & $1-10 \%$ & $0.1 \%-1 \%$ & \\
\hline nuclides: 5 & 2 & 12 & 3 \\
\hline${ }^{* 233} \mathrm{UTFH}$ & ${ }^{* 240} \mathrm{Pu} \mathrm{F}$ & ${ }^{* 232} \mathrm{Th} F H$ & ${ }^{252} \mathrm{Ct} S p$ \\
\hline${ }^{* 235} \mathrm{UTFH}$ & ${ }^{245} \mathrm{Cm} \mathrm{TF}$ & 234 & ${ }^{242} \mathrm{Cm} \mathrm{SP}$ \\
\hline${ }^{* 238} \mathrm{UTFH}$ & & ${ }^{236} U F$ & ${ }^{244} \mathrm{Cm} \mathrm{SP}$ \\
\hline${ }^{* 239} \mathrm{Pu} \mathrm{TF}$ & & ${ }^{237} \mathrm{~Np} T F$ & \\
\hline \multirow[t]{8}{*}{${ }^{2441} P u T F$} & & ${ }^{238} \mathrm{~Np} T F$ & \\
\hline & & ${ }^{238} \mathrm{Pu} T F$ & \\
\hline & & ${ }^{242} P u F$ & \\
\hline & & ${ }^{241} A m T F$ & \\
\hline & & ${ }^{242 m} \mathrm{Am} T F$ & \\
\hline & & ${ }^{243} \mathrm{Am} T F$ & \\
\hline & & ${ }^{243} \mathrm{Cm} \mathrm{TF}$ & \\
\hline & & ${ }^{244} \mathrm{Cm} \mathrm{TF}$ & \\
\hline
\end{tabular}

* Nuclides in UKFY1 and previous UK libraries.

$\mathrm{T}$ Thermal fission.

F Fast fission.

H 14Mev Fission.

Sp Spontaneous fission.

experimental cumulative yields and their uncertainties, produce uncertainties for all the cumulative yields.

- Produce an ENDF formatted file containing the data.

\subsection{Changes in the UKFY3.7 evaluation}

\subsubsection{New data}

The previous UK evaluation included a literature search complete up to 2000. A review was carried out in 2013 and ten important new references identified. In addition, a further reference was identified in 2016 for fast fission yields of neodymium isotopes that was also included. The totals of absolute, relative and "ratio of ratio" measurements in the old and new databases are shown in Table 2. The split of the new data between different fissioning systems are shown in Table 3. For the fast neutron spectra cases the new data refers to neodymium cumulative yields and the results of the statistical analysis for the affected Nd148 cumulative yields are shown in Table 4 with their uncertainties.

The improvements to the mass distributions are of two classes; firstly, where previously unmeasured yields are now available, and secondly, where new data of has altered the best estimate of a yield. The first class includes; thermal fission of $\mathrm{Np} 238$ where new data is now available between masses 74 and $85, \mathrm{Pu} 239$ where data for masses 80,82 and 130 have now been measured, and Cf249 where data is now available for masses 69 to 82 . For the second class, Fig. 1 shows the relative changes in the mass yields for the other thermal fission yields including new data. It should be noted that the new data significantly alters many of the values, especially in the valley and wings of the mass distribution.

\subsubsection{New modelling}

In the JEFF-3.1.1 (UKFY3.6A) evaluation the chain yields were modelled using the 5-Gaussian approximation
Table 2. Data items in the previous and current database.

\begin{tabular}{|c|c|c|c|c|}
\hline Dataset & Absolute & Relative & $\begin{array}{c}\text { "ratio } \\
\text { of } \\
\text { ratio" }\end{array}$ & Total \\
\hline UKFY3.6A & 11887 & 1352 & 1471 & 14710 \\
\hline UKFY3.7 & 12924 & 1442 & 1471 & 15837 \\
\hline
\end{tabular}

Table 3. Number of measured data items for fissioning systems with new data.

\begin{tabular}{|c|c|c|c|c|}
\hline $\begin{array}{c}\text { Neutron } \\
\text { spectra }\end{array}$ & $\begin{array}{c}\text { Fissioning } \\
\text { nuclide }\end{array}$ & UKFY3.6 & $\begin{array}{c}\text { New } \\
\text { data }\end{array}$ & UKFY3.7 \\
\hline Thermal & Th229 & 337 & 72 & 409 \\
\hline Thermal & U233 & 757 & 188 & 945 \\
\hline Thermal & U235 & 2390 & 151 & 2541 \\
\hline Thermal & Np238 & 115 & 63 & 178 \\
\hline Thermal & Pu239 & 861 & 225 & 1086 \\
\hline Thermal & Pu241 & 334 & 63 & 397 \\
\hline Thermal & Cm245 & 161 & 219 & 380 \\
\hline Thermal & Cf249 & 305 & 239 & 544 \\
\hline Fast & U235 & 724 & 5 & 729 \\
\hline Fast & Pu239 & 390 & 5 & 395 \\
\hline Fast & Pu241 & 111 & 5 & 116 \\
\hline
\end{tabular}

Table 4. The fast neutron spectra ${ }^{148} \mathrm{Nd}$ cumulative yields determined from measurements.

\begin{tabular}{|c|c|c|}
\hline Fissioning nuclide & UKFY3.7 & UKFY3.6A \\
\hline U235 & $1.677 \pm 0.020$ & $1.696 \pm 0.019$ \\
\hline Pu239 & $1.700 \pm 0.029$ & $1.699 \pm 0.025$ \\
\hline Pu241 & $1.943 \pm 0.041$ & $1.946 \pm 0.045$ \\
\hline
\end{tabular}

with the parameters fitted for each fissioning system where possible and the parameters were extrapolated for fissioning system with too little data to be fitted. Similarly, the Wahl Zp model [4] was used to fit systems with enough data and the model parameters were extrapolated for other systems.

Recently, a new fission model code, GEF ("GEneral description of Fission observables") has shown good agreement for mass and charge distributions against experiment [6]. A great advantage of the model is that it uses a standard set of selected and fitted parameters for predicting yields from the fission of isotopes of elements from thorium to californium, and at neutron energies from thermal up to and including second chance fission.

Following some initial comparisons between the UKFY3.6A database and the GEF predictions in the JEFF community it was decided to use the GEF model predictions for mass and charge distributions to replace the 5-Gaussian and Zp models.

The GEF model can also estimate isomeric splitting of independent yields but due to the lack of experimental data and the lack of an updated JEFF-3.3 draft decay data file (from which the models get the spins of the ground and isomeric states) it was decided to use the existing isomeric splitting in this evaluation. In addition, the JEFF3.1.1 decay data library is used throughout the evaluation and testing reported in this paper.

\section{Initial testing of UKFY3.7}

Fission product yields are important in many different types of calculations, but for the initial testing it was 

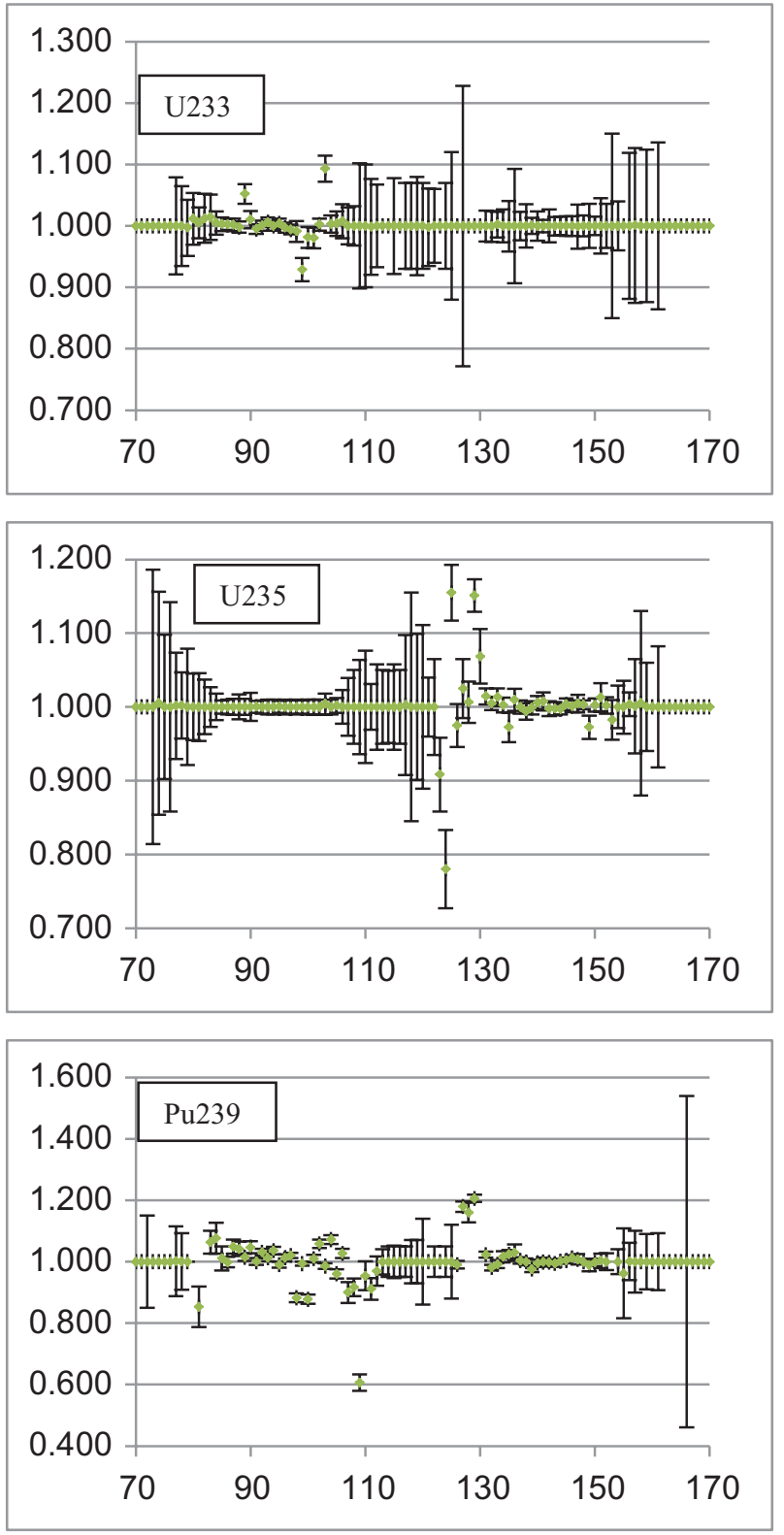
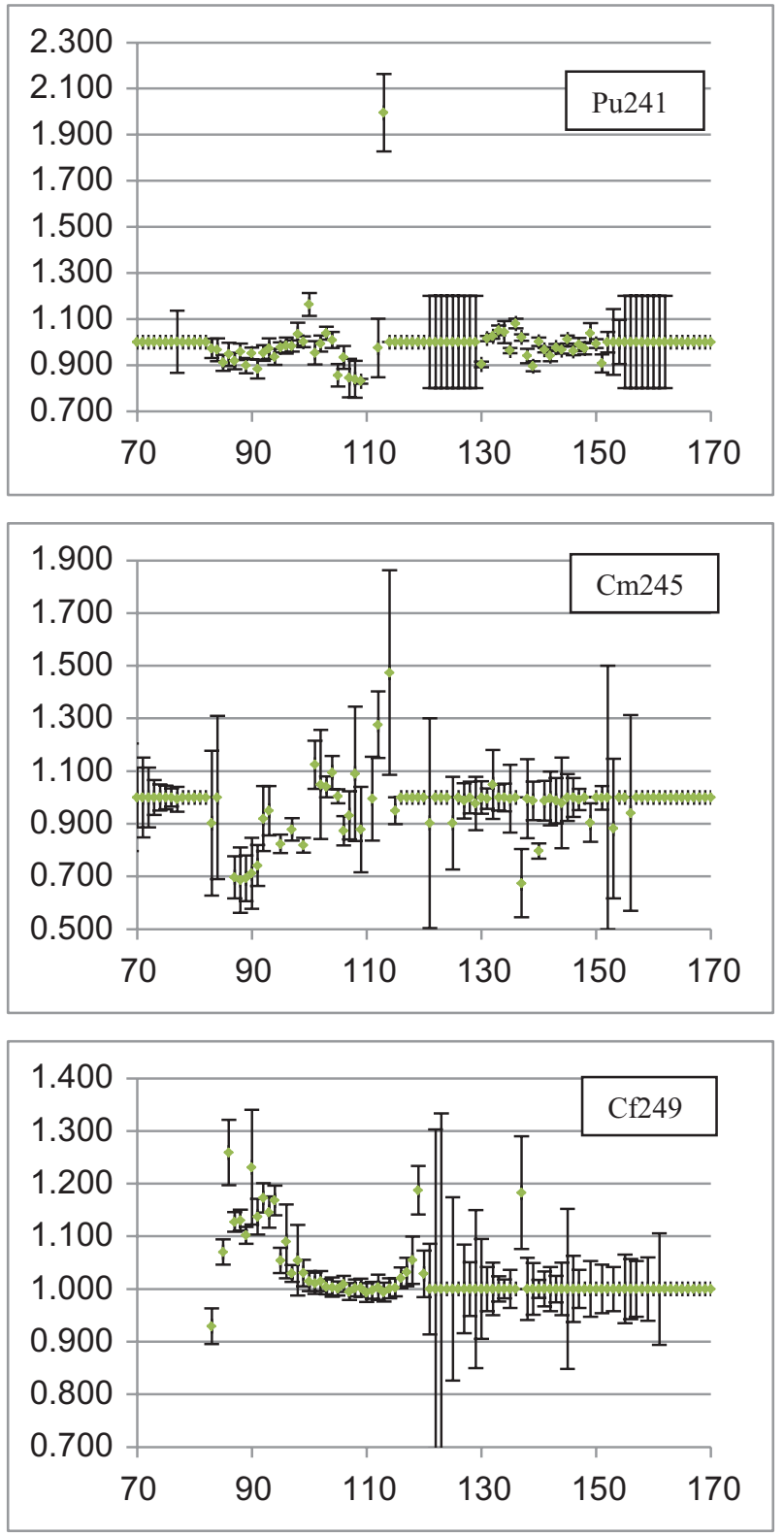

Figure 1. For nuclides with significant new data, the plots show changes to the mass yields determined from the UKFY3.7 database as a ratio to those determined previously in UKFY3.6A against the yield mass. The new uncertainties are shown to illustrate the accuracy.

decided to use three types of calculation; (i) the average number of delayed neutrons per fission, (ii) the decay heat following a single fission pulse and (iii) the decay heat measured from spent PWR fuel assemblies.

\subsection{Delayed neutron summation calculation}

The average number of delayed neutrons per fission $\left(\bar{v}_{d}\right)$ can be calculated using the cumulative yields of a fissioning system $\left(C_{i}\right)$ and the number of delayed neutrons emitted per decay of a nuclide i $\left(P_{n}^{i}\right)$ using

$$
\bar{v}_{d}=\sum_{i=0}^{n} C_{i} P_{n}^{i}
$$

The calculation results for UKFY3.7 and JEFF-3.1.1 fission product yields using the JEFF-3.1.1 decay data are shown in Table 5.

The variance of the $\bar{v}_{d}$ culations can be determined given the values and variances of the cumulative yields and the $P_{n}^{i}$ values using

$$
\sigma_{\bar{v}_{d}}^{2}=\sum_{i=0}^{n} \sigma_{C_{i}}^{2} P_{n}^{i^{2}}+\sum_{i=0}^{n} C_{i}^{2} \sigma p_{n}^{i^{2}}
$$

The delayed neutron emission is strongly dependent upon the yield distribution away from stability where delayed neutron emission is possible. In previous work [4] it was noted that the ratio of the calculation to experiment showed a trend with the mass of the fissioning nucleus. Although there are large uncertainties on the summation calculations it is noted that the UKFY3.7 calculations, given in Fig. 2, shows a scatter around about a ratio of 0.95 but showed no significant trend in nucleus mass. It is too early to decide if this slight improvement results from switching the previous JEFF-3.1.1 extrapolation of Zp model parameters to the more self-consistent GEF model, but at the very least the UKFY3.7 results are no worse than the previous JEFF-3.1.1 results. 
Table 5. Calculation of $\bar{v}_{d}$ using UKFY3.7 and JEFF-3.1.1.

\begin{tabular}{|c|c|c|c|c|}
\hline System & $\begin{array}{c}\text { Neutron } \\
\text { energy }\end{array}$ & UKFY3.7 & JEFF-3.1.1 & $\begin{array}{c}\bar{v}_{d} \text { from } \\
\text { experiment }[4]\end{array}$ \\
\hline Th232 & Fast & $4.081 \pm 0.184$ & $5.384 \pm 0.237$ & $5.47 \pm 0.12$ \\
\hline Th232 & $14 \mathrm{MeV}$ & $2.907 \pm 0.165$ & $3.056 \pm 0.211$ & $2.85 \pm 0.13$ \\
\hline U233 & Thermal & $0.738 \pm 0.062$ & $0.724 \pm 0.056$ & $0.664 \pm 0.018$ \\
\hline $\mathrm{U} 233$ & Fast & $0.757 \pm 0.063$ & $1.042 \pm 0.079$ & $0.729 \pm 0.019$ \\
\hline $\mathrm{U} 233$ & $14 \mathrm{MeV}$ & $0.411 \pm 0.040$ & $0.559 \pm 0.060$ & $0.422 \pm 0.025$ \\
\hline U234 & Fast & $1.079 \pm 0.082$ & $1.342 \pm 0.169$ & $1.06 \pm 0.12$ \\
\hline $\mathrm{U} 235$ & Thermal & $1.471 \pm 0.083$ & $1.477 \pm 0.079$ & $1.654 \pm 0.042$ \\
\hline $\mathrm{U} 235$ & Fast & $1.444 \pm 0.080$ & $1.698 \pm 0.087$ & $1.714 \pm 0.022$ \\
\hline $\mathrm{U} 235$ & $14 \mathrm{MeV}$ & $0.800 \pm 0.064$ & $0.934 \pm 0.071$ & $0.927 \pm 0.029$ \\
\hline U236 & Thermal & $2.487 \pm 0.118$ & & \\
\hline U236 & Fast & $1.933 \pm 0.111$ & $2.386 \pm 0.152$ & $2.31 \pm 0.26$ \\
\hline U238 & Fast & $3.535 \pm 0.131$ & $4.037 \pm 0.129$ & $4.51 \pm 0.061$ \\
\hline U238 & $14 \mathrm{MeV}$ & $2.295 \pm 0.093$ & $2.369 \pm 0.098$ & $2.73 \pm 0.08$ \\
\hline Np237 & Thermal & $1.303 \pm 0.083$ & $1.130 \pm 0.076$ & $1.07 \pm 0.10$ \\
\hline Np237 & Fast & $1.141 \pm$ & $1.169 \pm 0.064$ & $1.22 \pm 0.03$ \\
\hline Np238 & Thermal & $1.658 \pm 0.202$ & $1.419 \pm 0.135$ & \\
\hline Np238 & Fast & $1.783 \pm 0.188$ & $1.635 \pm 0.202$ & \\
\hline $\mathrm{Pu} 238$ & Thermal & $0.376 \pm 0.035$ & $0.319 \pm 0.038$ & $0.456 \pm 0.051$ \\
\hline $\mathrm{Pu} 238$ & Fast & $0.455 \pm$ & $0.483 \pm 0.067$ & $0.456 \pm 0.051$ \\
\hline $\mathrm{Pu} 239$ & Thermal & $0.628 \pm 0.043$ & $0.605 \pm 0.043$ & $0.624 \pm 0.024$ \\
\hline $\mathrm{Pu} 239$ & Fast & $0.605 \pm 0.044$ & $0.675 \pm 0.046$ & $0.664 \pm 0.013$ \\
\hline $\mathrm{Pu} 240$ & Fast & $0.809 \pm 0.054$ & $0.920 \pm 0.058$ & $0.96 \pm 0.11$ \\
\hline $\mathrm{Pu} 241$ & Thermal & $1.306 \pm$ & $1.232 \pm 0.058$ & $1.56 \pm 0.16$ \\
\hline $\mathrm{Pu} 241$ & Fast & $1.270 \pm 0.062$ & $1.290 \pm 0.067$ & $1.63 \pm 0.16$ \\
\hline $\mathrm{Pu} 242$ & $\mathrm{~F}$ & $1.679 \pm 0.081$ & $1.684 \pm 0.078$ & $2.28 \pm 0.25$ \\
\hline Am241 & Thermal & $0.488 \pm 0.043$ & $0.378 \pm 0.047$ & $0.44 \pm 0.05$ \\
\hline Am241 & Fast & $0.486 \pm 0.059$ & $0.414 \pm 0.055$ & $0.394 \pm 0.024$ \\
\hline Am242 & Thermal & $0.712 \pm 0.111$ & $0.582 \pm 0.106$ & $0.69 \pm 0.05$ \\
\hline Am242 & Fast & $0.714 \pm 0.048$ & $0.567 \pm 0.075$ & \\
\hline Am243 & Thermal & $1.038 \pm 0.063$ & $0.842 \pm 0.113$ & \\
\hline Am243 & Fast & $1.034 \pm$ & $0.816 \pm 0.099$ & \\
\hline $\mathrm{Cm} 242$ & Spont. & $0.115 \pm 0.026$ & $0.120 \pm 0.022$ & \\
\hline $\mathrm{Cm} 243$ & Thermal & $0.275 \pm 0.029$ & $0.221 \pm 0.043$ & \\
\hline $\mathrm{Cm} 243$ & Fast & $0.284 \pm 0.028$ & $0.212 \pm 0.030$ & \\
\hline $\mathrm{Cm} 244$ & Spont. & $0.331 \pm 0.044$ & $0.317 \pm 0.041$ & \\
\hline Cm244 & Thermal & $0.416 \pm 0.037$ & $0.326 \pm 0.055$ & \\
\hline $\mathrm{Cm} 244$ & Fast & $0.376 \pm 0.032$ & $0.334 \pm 0.042$ & \\
\hline $\mathrm{Cm} 245$ & Thermal & $0.570 \pm 0.044$ & $0.526 \pm 0.071$ & $0.59 \pm 0.04$ \\
\hline Cm245 & Fast & $0.591 \pm 0.042$ & $0.466 \pm 0.065$ & \\
\hline Cf252 & Spont. & $0.668 \pm 0.039$ & $0.585 \pm 0.030$ & $0.86 \pm 0.10$ \\
\hline
\end{tabular}

\subsection{Single fission pulse decay heat}

There exists many measurements of fission product decay heat from different nuclides following a short fission pulse. In this work, the standard benchmark for thermal fission of U235 and Pu239 [7], which were derived from the analysis of many experiments, were compare with summation calculations. The FISPIN inventory code [8] was used with the UKFY3.7 and JEFF-3.1.1 fission yield files and the JEFF-3.1.1 decay data to compare with these benchmarks. These results are shown in Figs. 3 and 4.

There is a small difference between these two sets of results, although only just outside the one standard deviation range. Below 10 seconds there is a small improvement, between 10 and 1000 seconds there is a slight worsening and at longer times there is little difference. These results suggest that the change between the previous Wahl Zp model and the new GEF model for these fissioning systems has little effect on the important

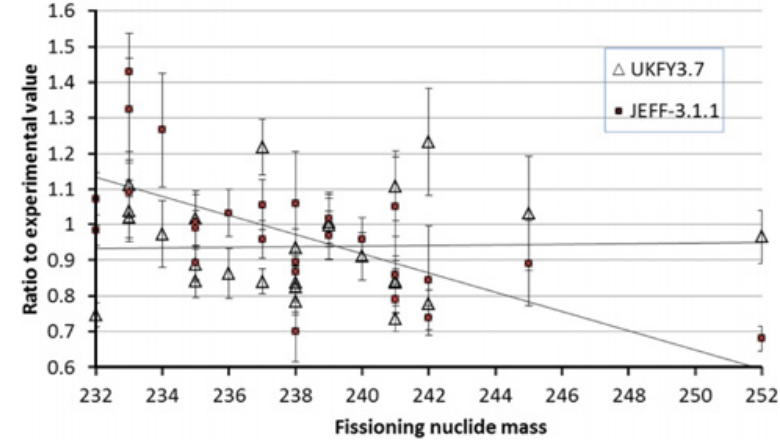

Figure 2. Plot of $\bar{v}_{d}$ calculation over experiment ratio using UKFY3.7 and JEFF-3.1.1 yields with JEFF-3.1.1 decay data shown against the fissioning nucleus mass. The lines are fitted to the two sets of results using $\mathrm{y}=\mathrm{ax}+\mathrm{b}$.

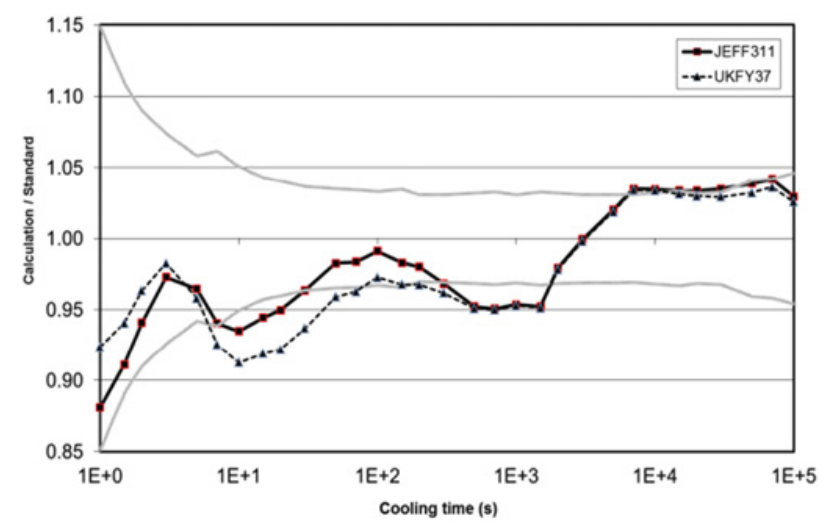

Figure 3. Plot of U235 decay heat against time following a single thermal neutron induced fission pulse using UKFY3.7 and JEFF-3.1.1 yields compared against the Tobias benchmark shown with a one standard deviation range [7].

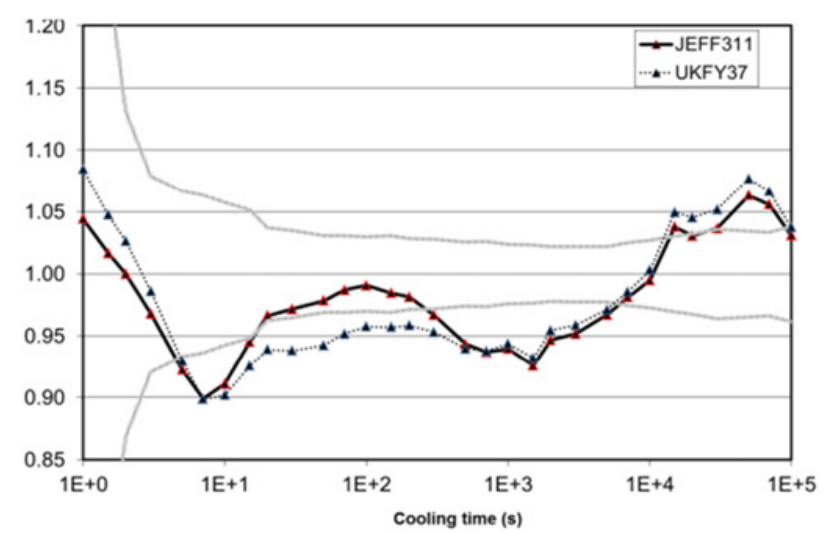

Figure 4. Plot of Pu239 decay heat following a single thermal neutron induced fission pulse using UKFY3.7 and JEFF-3.1.1 yields compared against the Tobias benchmark shown with a one standard deviation range [7].

nuclides for decay heat for these actinides. However, given that these systems have the most complete sets of measured yields this is not too surprising.

\subsection{PWR spent fuel assembly decay heat}

In previous work the agreement between measured decay heats from PWR assemblies at the CLAB facility [9] were compared to a series of inventory calculations using JEF-2.2 and JEFF-3.1.1 [10]. The modelling used in this 
Table 6. Description of the PWR assemblies measured at CLAB [9] and the calculation over experiment (C/E) ratio using simple inventory calculations using JEF-2.2, JEFF-3.1.1 and UKFY3.7. JEF-2.2 and JEFF-3.1.1 from [10].

\begin{tabular}{|c|c|c|c|c|c|c|c|c|c|c|c|c|c|c|c|c|}
\hline 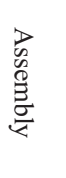 & 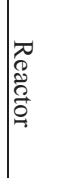 & 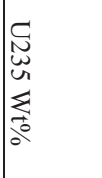 & 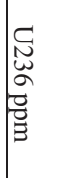 & 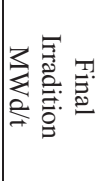 & 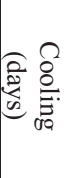 & 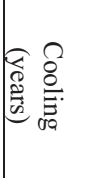 & 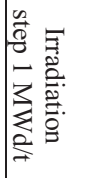 & 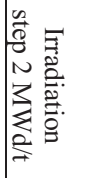 & 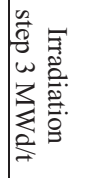 & 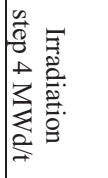 & 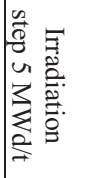 & 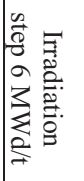 & 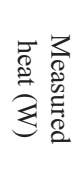 & 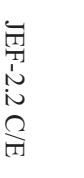 & م & 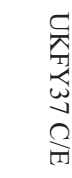 \\
\hline 0С9 & \multirow{20}{*}{ 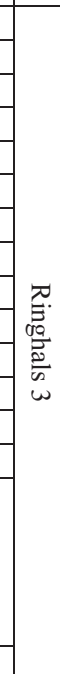 } & 3.101 & 150 & 38442 & 6551 & 17.94 & 9884 & 8192 & 10350 & 10016 & & & 491.2 & 1.000 & 0.991 & 0.993 \\
\hline 0E2 & & 3.103 & 150 & 41628 & 5823 & 15.94 & 7496 & 13034 & 11308 & 9790 & & & 587.9 & 0.974 & 0.967 & 0.970 \\
\hline 0E6 & & 3.103 & 150 & 35993 & 5829 & 15.96 & 12490 & 13031 & 10472 & & & & 487.7 & 0.986 & 0.975 & 0.977 \\
\hline $1 \mathrm{C} 2$ & & 3.101 & 150 & 33318 & 6559 & 17.96 & 6249 & 5019 & 11509 & 10541 & & & 417.7 & 0.999 & 0.988 & 0.990 \\
\hline $1 \mathrm{C} 5$ & & 3.101 & 150 & 38484 & 6593 & 18.05 & 9884 & 8102 & 10411 & 10087 & & & 499.2 & 0.983 & 0.974 & 0.977 \\
\hline 1E5 & & 3.103 & 150 & 34638 & 5818 & 15.93 & 10556 & 13134 & 10948 & & & & 468.8 & 0.986 & 0.975 & 0.978 \\
\hline $2 \mathrm{~A} 5$ & & 2.1 & 150 & 20107 & 7297 & 19.98 & 12228 & 7879 & & & & & 233.7 & 1.015 & 1.000 & 1.002 \\
\hline $2 \mathrm{C} 2$ & & 3.101 & 150 & 36577 & 6550 & 17.93 & 7783 & 8345 & 9932 & 10517 & & & 466.5 & 0.998 & 0.988 & 0.990 \\
\hline $3 \mathrm{C} 1$ & & 3.101 & 150 & 36572 & 6545 & 17.92 & 7783 & 8341 & 9931 & 10517 & & & 470.2 & 0.988 & 0.978 & 0.980 \\
\hline $3 \mathrm{C} 4$ & & 3.101 & 150 & 38447 & 6544 & 17.92 & 9884 & 8192 & 10354 & 10017 & & & 497.3 & 0.984 & 0.976 & 0.978 \\
\hline $3 \mathrm{C} 5$ & & 3.101 & 150 & 38373 & 6543 & 17.91 & 9884 & 8113 & 10343 & 10033 & & & 501.4 & 0.980 & 0.971 & 0.974 \\
\hline $3 \mathrm{C} 9$ & & 3.101 & 150 & 36560 & 6552 & 17.94 & 7783 & 8377 & 9876 & 10524 & & & 468.4 & 0.992 & 0.982 & 0.985 \\
\hline $4 \mathrm{C} 4$ & & 3.101 & 150 & 33333 & 6572 & 17.99 & 6249 & 4991 & 11030 & 11063 & & & 422 & 0.989 & 0.978 & 0.980 \\
\hline $4 \mathrm{C} 7$ & & 3.101 & 150 & 38370 & 6549 & 17.93 & 9884 & 8101 & 10347 & 10038 & & & 498.7 & 0.983 & 0.975 & 0.977 \\
\hline \multirow{5}{*}{$5 \mathrm{~A} 3$} & & \multirow{5}{*}{2.1} & \multirow{5}{*}{150} & \multirow{5}{*}{19699} & 6972 & 19.09 & \multirow{5}{*}{11696} & \multirow{5}{*}{8003} & & & & & 237.7 & 0.991 & 0.976 & 0.978 \\
\hline & & & & & 6975 & 19.10 & & & & & & & 236.6 & 0.996 & 0.981 & 0.982 \\
\hline & & & & & 6977 & 19.10 & & & & & & & 243.4 & 0.968 & 0.953 & 0.955 \\
\hline & & & & & 7291 & 19.96 & & & & & & & 230.9 & 1.005 & 0.990 & 0.991 \\
\hline & & & & & 7304 & 20.00 & & & & & & & 230.2 & 1.007 & 0.992 & 0.994 \\
\hline $5 \mathrm{~F} 2$ & & 3.404 & 150 & 47308 & 4724 & 12.93 & 13475 & 6922 & 10337 & 8930 & 7644 & & 714 & 0.976 & 0.971 & 0.974 \\
\hline C01 & \multirow{23}{*}{ 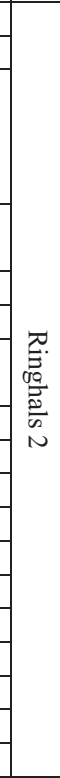 } & 3.095 & 150 & 36688 & 8468 & 23.18 & 11247 & 9403 & 7569 & 8469 & & & 415.7 & 1.006 & 0.998 & 0.999 \\
\hline $\mathrm{C} 12$ & & 3.095 & 150 & 36385 & 8403 & 23.01 & 11247 & 9318 & 7390 & 8430 & & & 410.3 & 1.009 & 1.000 & 1.001 \\
\hline \multirow{4}{*}{ C20 } & & \multirow{4}{*}{3.095} & \multirow{4}{*}{150} & \multirow{4}{*}{35720} & 6950 & 19.03 & \multirow{4}{*}{11247} & \multirow{4}{*}{9377} & \multirow{4}{*}{7454} & \multirow{4}{*}{7642} & & & 415.8 & 1.034 & 1.026 & 1.028 \\
\hline & & & & & 6951 & 19.03 & & & & & & & 426.1 & 1.009 & 1.001 & 1.003 \\
\hline & & & & & 6952 & 19.03 & & & & & & & 428.9 & 1.003 & 0.994 & 0.996 \\
\hline & & & & & 6959 & 19.05 & & & & & & & 435.6 & 0.987 & 0.979 & 0.981 \\
\hline C12 & & 2005 & 150 & 25630 & 5803 & 15.89 & 16565 & 7610 & 8126 & 2320 & & & 442.3 & 0.991 & 0.983 & 0.985 \\
\hline 442 & & 3.095 & 150 & 30539 & 5804 & 15.89 & 10563 & 1619 & 8126 & 3329 & & & 448.4 & 0.978 & 0.970 & 0.972 \\
\hline D27 & & 3.252 & 150 & 39676 & 7669 & 21.00 & 9510 & 12889 & 9267 & 8010 & & & 456 & 0.991 & 0.983 & 0.985 \\
\hline D38 & & 3.252 & 150 & 39403 & 8005 & 21.92 & 6367 & 9331 & 7358 & 8701 & 7646 & & 442.4 & 1.001 & 0.992 & 0.994 \\
\hline $\mathrm{F} 38$ & & 3199 & 150 & 33973 & 7999 & 21.90 & 7568 & 8458 & 9879 & 8068 & & & 376.3 & 0.995 & 0.984 & 0.986 \\
\hline EJ0 & & & & & 8000 & 21.90 & & & & & & & 374.3 & 1.000 & 0.989 & 0.991 \\
\hline E40 & & 3.199 & 150 & 34339 & 8075 & 22.11 & 7705 & 7249 & 10655 & 8730 & & & 381.2 & 0.992 & 0.982 & 0.983 \\
\hline F14 & & 3.197 & 150 & 34009 & 7722 & 21.14 & 5069 & 10755 & 9898 & 8287 & & & 381.8 & 1.003 & 0.992 & 0.994 \\
\hline F21 & & 3.197 & 150 & 36273 & 7376 & 20.19 & 4767 & 6317 & 10046 & 8255 & 6888 & & 420.9 & 0.992 & 0.982 & 0.984 \\
\hline F25 & & 3.197 & 150 & 35352 & 7725 & 21.15 & 8307 & 10749 & 8316 & 7980 & & & 396.7 & 1.011 & 1.000 & 1.002 \\
\hline F32 & & 3.197 & 150 & 50962 & 5860 & 16.04 & 10553 & 10609 & 8391 & 7761 & 6629 & 7019 & 692 & 0.994 & 0.990 & 0.994 \\
\hline G11 & & 3.188 & 150 & 35463 & 6990 & 19.14 & 6890 & 10422 & 7868 & 6943 & 3340 & & 416.3 & 0.992 & 0.982 & 0.984 \\
\hline G23 & & 3.206 & 150 & 35633 & 6984 & 19.12 & 10268 & 10035 & 7618 & 7712 & & & 420.7 & 0.996 & 0.986 & 0.988 \\
\hline I09 & & 3.203 & 150 & 40188 & 5849 & 16.01 & 6727 & 8950 & 9065 & 7568 & 7878 & & 507.9 & 1.011 & 1.003 & 1.006 \\
\hline I20 & & 3.203 & 150 & 34313 & 6588 & 18.04 & 8300 & 9010 & 9108 & 7895 & & & 403.5 & 0.987 & 0.976 & 0.978 \\
\hline I24 & & 3.203 & 150 & 34294 & 6601 & 18.07 & 8245 & 8967 & 9144 & 7938 & & & 410 & 0.983 & 0.972 & 0.974 \\
\hline I25 & & 3.203 & 150 & 36859 & 6198 & 16.97 & 5207 & 4991 & 9803 & 8998 & 7860 & & 445.8 & 1.000 & 1.000 & 1.003 \\
\hline & & & ean an & Standar & Devia & tion of $\mathrm{C}$ & /E values & & & & Ringl & als 3 & & $\begin{array}{c}0.990 \\
\pm \\
0.012\end{array}$ & $\begin{array}{c}0.979 \\
\pm \\
0.010\end{array}$ & $\begin{array}{c}0.981 \\
\pm \\
0.010\end{array}$ \\
\hline & & & & & & & & & & & Ringl & als 2 & & $\begin{array}{c}0.999 \\
\pm \\
0.012\end{array}$ & $\begin{array}{c}0.990 \\
\pm \\
0.012\end{array}$ & $\begin{array}{c}0.992 \\
\pm \\
0.012\end{array}$ \\
\hline & & & & & & & & & & & Coml & jined & & $\begin{array}{c}0.995 \\
\pm \\
0.013\end{array}$ & $\begin{array}{c}0.985 \\
\pm \\
0.012\end{array}$ & $\begin{array}{c}0.987 \\
\pm \\
0.012\end{array}$ \\
\hline
\end{tabular}

work was rudimentary but it was felt adequate as a sanity check prior to JEFF-3.3 decay data becoming available and JEFF-3.3 libraries becoming available for reactor modelling codes.

The results in Table 6 show an agreement with experiment that is comparable to previous libraries and within the $2 \%$ uncertainty estimate on the measured decay heat.

\subsection{Conclusions}

The results from these three initial tests show either a slight improvement or no significant changes. Further testing needs to be done and the improvements when combined with the new JEFF-3.3 decay data, when ready, will need to be assessed but the current study shows no initial concerns precluding further testing. 


\section{Future developments}

\subsection{Uncertainties on engineering quantities}

It is an important requirement for future development of nuclear technology that rigorous uncertainties on important engineering quantities need to be calculable. As fission yields are highly correlated it is necessary to provide the methods and covariance information to handle uncertainties for fission product related inventories and aggregate properties such as decay heat and radiative emissions.

There are several possible approaches to generate covariance data being considered based upon; (i) minimisation of physical constraints, (ii) perturbation of the model parameters underlying the yield distributions, (iii) LSQ or maximum likelihood methods using both an underlying model of fission and experimental data, examples can be found in the $[6,11-14]$ and the references therein. However, as the cumulative yields can be directly calculated from the independent yields using decay data, it is also possible to determine the covariance terms iteratively from the experimental data in this evaluation [15]. Now that the new evaluation is complete it is intended to test this iterative data driven method and collaborate with colleagues in the ENDF and JEFF communities to study the effectiveness of the other techniques.

\subsection{New measurement types}

The current evaluation process only considers measurements of specific mass, charge, and where relevant isomeric state, yields. Measurements that have a resolution of more than one mass or charge unit have been ignored, but these contain much useful information. In future evaluations it is hoped to generalize the analysis method so that these data can be included.

\subsection{Yields against neutron energy}

The current evaluations are based upon representative neutron spectra; "thermal", "fast" and "14 MeV". The new GEF model allows the easy production of many specific energy yield sets for a nuclide. It is hoped that future evaluations will be able to use the GEF method with experimental data to generate energy dependent yield sets with sufficient resolution to be useful in calculation, although a computation infrastructure, such as that used for neutron cross-sections, would need to be developed to use such data.

The author would like to thank Drs. Cabellos, Diez, Hambsch, Koning, Noguere, Pigni, Rochman, Schmidt, Serot and members of the JEFF and WPEC communities for useful discussions. Dr. Frost for entering the new fission yield data into the database and Dr. Mountford for extracting the GEF/GEFY model data for use in the evaluation. This work was funded by the NNL Research and Development fund and the EURATOM FP7 project CHANDA (FP7- 605203).

\section{References}

[1] E.A.C. Crouch, Atomic Data and Nuclear Data Tables, 19 (1977)

[2] J. Banai and M.F. James, (1987) Unpublished

[3] M.F. James, R.W. Mills, D.R. Weaver, Prog. Nucl. Energy 26, 1-29 (1991)

[4] R.W. Mills, "Fission product yield evaluation", Thesis, The University of Birmingham, UK (1995)

[5] M.A. Kellett, O. Bersillon, R.W. Mills, "The JEFF3.1/-3.1.1 Radioactive Decay Data and Fission Yields Sub-libraries", JEFF report 20, OECD, ISBN 97892-64-99087-6 (2009)

[6] K-H Schmidt, B. Jurado, C. Amouroux and C. Schmitt. Nuclear Data Sheets 131, 107-221 (2016)

[7] A.Tobias, CEGB report, RD-B-6210-R89 (1989)

[8] R.F. Burstal, UK Atomic Energy Authority report ND-R-328 (R) (1979)

[9] Svensk Kärnbränslehantering AB, "Measurements of decay heat in spent nuclear fuel at the Swedish interim storage facility, Clab", SKB Report R-05-62, ISSN 1402-3091 (2006)

[10] R.W. Mills, C.H. Zimmerman, C Shearer, "Validation of PWR spent fuel decay heat considering new SKB measurements", NEMEA-5 Neutron measurements, Evaluations and Applications -Nuclear data for sustainable nuclear energy; Proceedings of the CANDIDE workshop 27-29 October 2008

[11] D. Rochman, O. Leray, A. Vasiliev, H. Ferroukhi, A.J. Koning, M. Fleming and J.C. Sublet. Annals of Nuclear Energy 95, 125 (2016)

[12] N. Terranova, O. Serot, P. Archier, C. De Saint Jean, and M. Sumini. Nuclear Data Sheets 123, 225-230 (2015)

[13] L. Fiorito, A. Stankovskiy, G. Van den Eynde, C.J. Diez, O. Cabellos, P.E. Labeau, Annals of Nuclear Energy 88, 12-23 (2016)

[14] M.T. Pigni, I.C. Gauld, M.L. Williams, F. Havluj, D. Wiarda, and G. Ilas. "Applications of decay data and fission product yield covariance matrices in uncertainty quantification on decay heat" OECD/NEA WPEC Subgroup-37 meeting, 2013

[15] RW Mills, Nuclear Data Sheets 118, 484-487 (2014) 